Revue d'histoire de l'Amérique française

FB. REVUE D.HISTOIRE DE L'AMÉRIQUE FRANÇAISE

\title{
Le climat marial en Nouvelle-France à l'arrivée de Mgr de Saint-Vallier
}

\section{Hector Bibeau}

Volume 22, numéro 3, décembre 1968

URI : https://id.erudit.org/iderudit/302799ar

DOI : https://doi.org/10.7202/302799ar

Aller au sommaire du numéro

Éditeur(s)

Institut d'histoire de l'Amérique française

ISSN

0035-2357 (imprimé)

1492-1383 (numérique)

Découvrir la revue

Citer cet article

Bibeau, H. (1968). Le climat marial en Nouvelle-France à l'arrivée de Mgr de Saint-Vallier. Revue d'histoire de l'Amérique française, 22(3), 415-428.

https://doi.org/10.7202/302799ar d'utilisation que vous pouvez consulter en ligne.

https://apropos.erudit.org/fr/usagers/politique-dutilisation/ 


\section{LE CLIMAT * MARIAL EN NOUVELLE-FRANCE À L'ARRIVÉE DE MGR DE SAINT.VALLIER **}

Comme la vieille France, la nouvelle connut elle aussi sa ferveur mariale. Elle est née au moment où les grands courants de pensée commençaient de faire sillage. Le fait est par ailleurs assez frappant pour que, sur l'appui d'un simple coup d'œil, l'historien contemporain s'oblige de le noter. "La graduelle histoire du culte marial en Nouvelle-France" ${ }^{1}$ apparaît, selon Gustave Lanctot, dès les origines de la colonie. C'est aussi le sentiment des témoins de l'époque qui suit immédiatement celle que nous étudions. En 1741 , le sulpicien Clément Pagès commence la relation de son voyage en Canada par ces mots: "Je me suis mis sous la protection de la Sainte Vierge qui est singulièrement honorée dans ce pays." ${ }^{2}$ Le voyageur Kalm remarque, en 1748, que "la Vierge paraît plus honorée au Canada que Dieu même" 3 .

Postérieurs à la période de Mgr de Saint-Vallier, ces deux témoignages ont ceci de commun: ils indiquent, chacun à leur

* Souci de précision: le mot 'climat' veut souligner notre préoccupation ici; nous chercherons plus les faits que les idées. A cette 'époque d'artisanat' il n'y a guère de pensée autonome. Il est plus exact de parler d'artisans qui véhiculent - et filtrent — des idées mariales ayant cours dans la mère-patrie.

** Extrait d'une thèse intitulée: "La pensée mariale de Mgr de SaintVallier", pour l'obtention d'un diplôme d'études supérieures en histoire, Université Laval, 1966.

1 Voir son livre Une Nouvelle-France inconnue, 21-54; il étudie spécialement Le culte marial en Nouvelle-France.

2 Clément Pagès (1715-1769), Relation d'un voyage de Paris au Canada - 14 octobre 1741, cité par A. Godbout, o.f.m., Les Récollets apôtres de Marie sous le Régime français, dans Soc. can. hist. Egl., rapport 1953-1954: 13.

3 Kalm, Voyage en Amérique du Nord. Cité par A. Godbout, ibid.: 22. Rappelons que Pierre Kalm (1715-1779) est un botaniste suédois, de religion luthérienne. Des trois volumes qu'il a rédigés au retour de ce voyage, seul le 3ème traite du Canada. 
façon, un état de fait qui - on peut le présumer - a pris racine avant. Déjà en 1651, la Relation mentionne qu'aux TroisRivières: ${ }^{4}$

[...] il y avait un petit oratoire en chaque maison; l'un était dédié à Notre-Dame de Lorette, l'autre à Notre-Dame de Liesse, les autres à Notre-Dame des Vertus, de bon Secours, de bonne Nouvelle, de la Victoire, et à quantité d'autres titres sous lesquels on honore la Sainte Vierge en divers lieux de la chrétienté. C'était une dévotion ordinaire à ces pauvres habitants d'aller visiter ces petits oratoires en divers jours de la semaine, principalement les samedis, que le concours y était plus grand, et en chaque maison matin et soir, tout le monde se rassemblait pour y faire les prières en commun, et l'examen de leur conscience, et pour y dire les litanies de la très sainte Vierge: le chef de famille étant d'ordinaire celui qui faisait les prières, et auquel tous les autres répondaient [...]

Il n'apparaît pas que nous soyions en face d'une ferveur particulière et momentanée. Mgr de Saint-Vallier lui-même passe un aveu, plus discret, à son retour en France en 1687. Décrivant "l'attitude des Français qui composent la Colonie" il note: 5

[...] dans les habitations qui sont les plus éloignées des Paroisses [...]. Chaque maison est une petite Communauté bien réglée, où l'on fait la prière en commun soir et matin, où l'on récite le chapelet, où l'on a la pratique des examens particuliers avant les repas, et où les pères et mères de familles suppléent au défaut de Prêtres [...]

Une confrontation sommaire de ces témoignages permet l'hypothèse suivante: à l'arrivée du successeur de Mgr de Laval, il y avait une tradition mariale en Nouvelle-France. Il reste à la vérifier par les faits, à préciser l'action des chefs de file et les thèmes qu'ils ont fait valoir.

4R. G. Thwaites, The Jesuit Relations and Allied Documents [...], XXXVI: 166 .

5 Estat présent de l'Eglise et de la Colonie française dans la NouvelleFrance, par M. l'Evêque de Québec, dans H. Têtu et C.-O. Gagnon, Mandements Lettres pastorales et Circulaires des Evêques de Québec, I: 252-253. Nous citerons désormais: Estat présent et Mandements des Evêques. 
Semble-t-il, les circonstances se rencontrent pour rendre le climat spirituel favorable au développement d'un culte marial assez particulier. L'homme n'y est certes pas absent mais son action prend le rythme du pays à construire. Comme on n'a guère le temps de ratiociner, ce sont avant tous les gestes pratiques qui vont donner l'élan à une vie mariale intense. S'il y a des mystiques - et il y en eût - ce sont des apôtres; ils ont imprimé cette vie de leurs couleurs particulières. Autre caractéristique du milieu, il n'y a pas de clivage social, du moins à l'époque où nous sommes. Tous, militants d'église et colons liés par un commun labeur et convertis indigènes, communient aux mêmes gestes: l'osmose spirituelle est permanente.

Ces gestes collectifs mettent en relief une commune confiance en Marie. Plusieurs trouvent écho dans les Relations. Certains ont une portée d'appel plus limitée, comme cette coutume établie en 1633 par Champlain de faire 'tinter' au canon du fort les trois temps de l'Angelus "au commencement, au milieu et à la fin du jour" ". Il en est de plus spectaculaires par leur déploiement extérieur ${ }^{7}$, d'autres qui témoignent d'une vénération plus intérieure, telle l'érection en 1633 de la chapelle votive de NotreDame-de-la-Recouvrance dans laquelle les Jésuites feront déposer "une image en relief de Marie" 8 . Ce n'est pas le seul ex-voto du genre. La piété populaire et la reconnaissance de Sœur Bourgeois édifient en 1659, au flanc du Mont-Royal, la première chapelle commémorative de Notre-Dame-de-Bon-Secours ${ }^{9}$ qui devient un lieu de pèlerinage très fréquenté ${ }^{10}$. Un autre sanctuaire

${ }_{6}$ Relation de 1634, dans Thwaites, op. cit., VI: 102. Cette coutume de l'Angelus était toute récente en France: l'usuelle pratique en est généralisée après 1600 .

${ }^{7}$ Les Relations de 1637 et de 1639 , entre autres, contiennent quelques descriptions hautes en couleurs des processions populaires, avec la participation des Indiens, pour célébrer les fêtes de l'Immaculée-Conception et de I'Assomption. Voir Thwaites, op. cit., XI : 66; XV : 226.

8 Journal des Jésuites: Catalogue des Bienfaiteurs de N.-Dame de Recouvrance, dans Thwaites, op. cit., XLII : 268.

9 AHDM, Annales de Sœur Morin: 70 (74). Elle parle d" "un petit bâtiment de bois mais si dévôt que le peuple y allait comme à un asile assuré pour tous ses besoins [...]".

10 Ibid.: 71 (75). Après avoir dit "la confiance des peuples qui est grande envers Notre Dame de Bon-Secours" l'annaliste ajoute: “C'est la promenade des personnes dévotes de la ville qui vont tous les soirs en 
marial réputé est celui de Notre-Dame-de-Lorette: "C'est un lieu de pèlerinage pour les Français qui viennent de fort loin et en grand nombre pour y faire leurs dévotions et pour y demander des grâces à Dieu par l'intercession de la Sainte Vierge","11 lit-on dans une relation du temps. D'autres chroniques relatent avec moult détails le recours collectif à la Vierge, par la colonie entière, à l'occasion de la première invasion anglaise de $1690^{12}$. Le sentiment populaire fut commun à ce moment que la victoire était due à une intervention de la Vierge. Dans un mémoire particulier rédigé sous le coup de l'événement on peut lire: ${ }^{13}$

[...] l'on fit ensuite une procession solennelle en l'honneur de la Vierge Patronne du païs [...]. On a institué a perpétuité une feste soubs le nom de Nostre Dame des Victoires [sic], et l'Eglise qu'on a commencée à la Basse Ville est dédiée soubs le mesme nom pour être une marque éternelle de la protection que nous avons reçue du ciel dans cette attaque subite $[\ldots]$.

Répondant sans doute à une pression populaire, $\mathrm{Mgr}$ de Saint-Vallier ajoutera au calendrier liturgique la fête de NotreDame de la Victoire ${ }^{14}$ qui deviendra - à la suite de circonstances analogues en 1711 - Notre-Dame des Victoires.

A la rigueur cette dévotion-recours peut n'être qu'épisodique, voire superficielle. La greffe semble avoir poussé plus loin ses

pèlerinage et il y a peu de bons catholiques qui ne fassent des vœux et offrandes à cette chapelle, dans tous les périls où ils se trouvent, de tous les endroits du Canada qui est bien grand."

11 AAQ, Eglise du Canada, IV: 54.

12 En Acadie: une relation du P. Thury, dans J. Blanchet, Collection de Manuscrits [...], I: 464ss.

A Québec: Relation de la défaite des Anglais à Québec, signée Michel Germain de Couvert, dans AAQ, Eglise du Canada, IV: 56-61.

A Montréal: le 16 octobre 1690, des marchands font vœu d'honorer la Vierge après la victoire; voir ASSM, Registre de la Fabrique de Montréal (1657-1794): 37.

13 Relation de ce qui s'est passé de plus remarquable en Canada, depuis le départ des vaisseaux au mois de novembre, 1689, jusqu'au mois de novembre, 1690, Signée Monseignat. Dans J. Blanchet, op. cit.: 482-531. Citation: 530 .

14 Le mandement d'institution est daté du 19 septembre 1694. Voir Mandements des Evêques, I: 342; aussi la liste des fêtes observées dans le diocèse de Québec, ibid.: 335-336. 
racines, si l'on songe que de la petite trentaine de cellules paroissiales, en voie d'organisation en 1685 , près de la moitié sont placées sous un vocable marial et ainsi reliées d'assez près au mystère de Marie ${ }^{15}$. Mais - toujours au plan des faits combien plus significative encore et plus lourde en ses effets fut la curieuse fondation de Ville-Marie ! Dans le climat pieux de l'époque elle était un coup droit pour enfoncer la tendance déjà prise. Si l'élan a perdu de sa vivacité à l'arrivée de $\mathrm{Mgr}$ de Saint-Vallier, il a tout de même assez duré pour permettre aux fondateurs de Ville-Marie d'affermir un idéal religieux populaire fortement teinté de dévotion mariale.

Se pourrait-il que cet avant-poste soit resté un haut-lieu spirituel fermé ? Nous connaissons pour l'avoir déjà signalée la ferveur des habitants du poste trifluvien. Un coup de sonde du côté des associations pieuses - elles se multiplient durant les années 1650 - révèle que le pli n'est pas particulier; les aspirations à une vie mariale plus poussée y sont manifestes. Une "requête des habitants du Canada", adressée vraisemblablement en $1648{ }^{18}$ au "Reverend Père Prieur des Jacobins du Couvent de Sainct Jacques" demande: ${ }^{17}$
[...] les Habitants de la Nouvelle france demeurans es habitations de Quebec, les Trois Rivieres Et Mont- real disans que leur Pays ayant esté consacré et mis soubs la protection de la Saincte Vierge et de Sainct Joseph [...] Il vous Plaise de vostre grace de per- mettre l'establissement de la d-Confrerie du Sainct

15 "L'état des cures de Canada fait en 1686" permet d'en dénombrer 36. AAQ, Eglise du Canada, III: 15 . Un relevé sommaire des vocables indique comme dédiées à l'IMMACULÉE-CONCEPTION la cathédrale de Québec et les églises de Ville-Marie, des Trois-Rivières, de Saint-Ours; à la NATIVITÉ celle de Beauport, à la Présentation celle de Champlain; à la Visitation celles de Chateau-Richer, de l'Ange Gardien et de Notre-Dame-de-Foy; à l'Assomption celles de la Rivière-Ouelle et de Port-Royal; il y a en plus Notre-Dame-de-Lorette et Notre-Dame-des-Anges. Voir dans Mandements des Evêques, I: 115-118 un Plan général de l'état présent des missions du Canada fait en l'année 1683, par Mgr de Laval.

16 La lettre patente, signée par le Père Charles Thébault, pour l'érection de la confrérie du Rosaire à Québec est datée du 28 mai 1649; elle est contresignée par le Père Jérôme Lalemant de Québec, le 23 octobre 1650. APNDQ, Répertoire, Série III, carton 1: nos 20-21.

17 APNDQ, ibid. : no 19. 
Rosaire dans leurs habitations de Quebec, les Trois Rivieres et Montreal, en la Nouvelle france pour d'autant plus y accroistre la dévotion de la Saincte Vierge [...].

Chaînon initial peut-on dire d'un embrigadement plus ample, sous l'action des curés-missionnaires, après les années $1656{ }^{18}$. Le mouvement est bouclé par l'établissement de la confrérie de la Sainte-Famille qui, de toutes, a eu l'influence la plus durable au sein des familles ${ }^{19}$; elle est à l'origine d'une spiritualité familiale ${ }^{20}$ où la Vierge tenait le premier rôle. Et signe qu'il n'y a pas de clivage religieux - est-il sans intérêt de le noter les affiliés se recrutent dans tous les groupements naturels: administrateurs, clergé, familles religieuses, colons, indigènes ${ }^{21}$.

Ce ne sont là que des indices - encore externes - pour identifier une tradition mariale en Nouvelle-France. Son fondement est beaucoup mieux assuré s'il est possible de dégager

18 La confrérie du Rosaire est effectivement fondée à Québec le 1er octobre 1656 par le Père Joseph Poncet, APNDQ, Série II, no 35: Livre du St Rosaire; à Montréal le 3 novembre suivant par le Père Bijart, APNDQ, Série III, carton 1: no 23. La confrérie du Mont-Carmel dont la lettre d'érection (10 mai 1647) n'a pu être mise en application "en raison de l'incommodité de l'Eglise qui n'était pas en estat pour pratiquer les exercices de la dite confrérie" est fondée à Québec le 1er novembre 1656, APNDQ, Série II, no 26: Registre de la Confrérie du Mont-Carmel; Série III, carton 1: no 29; à Montréal le 26 août 1694, AHDM, Annales, III : 82. Depuis 1654, les Hurons de l'Ile d'Orléans ont leur 'congrégation' mariale fondée par le Père Joseph-Marie Chaumonot, Relation de 1654, dans Thwaites, op. cit., XLI: 146ss. Le 14 février 1657 le Père Poncet fonde celle de Québec, Journal des Jésuites, édition Laverdière et Casgrain, 206.

19 Elle commence à Ville-Marie en 1663 par les soins de Sœur Bourgeois, E.-M. Faillon, Vie de la Sour Bourgeois [...], I: 163. Les lettres patentes, - l'original est aux APNDQ, Série III, carton I: no 16 - de Mgr Laval sont datées du 10 mars 1665.

20 Cette fraternité, fortement structurée, avait des règlements très détaillés, des prières, des méthodes d'oraison, un office liturgique, un catéchisme, un catalogue d'indulgences. Voir le tout aux APNDQ, Série II: nos 28, 31, 31A ; Série III, carton 1: nos 17 et 26 . Les règlements se trouvent dans Mandements des Evếques, I: 56-66.

21 Dans les registres de la Confrérie de la Sainte-Famille de la paroisse Notre-Dame de Québec figurent les noms de Mgr de Laval et de l'intendant Pierre Duchesnau inscrits le 13 novembre 1667, ceux de 22 Hospitalières de l'Hôtel-Dieu de Québec et de 25 Ursulines pour l'année 1672; figurent également des noms de Hurons. Le Registre de la Confrérie du Rosaire de la même paroisse porte des noms de prêtres, de diacres, d'ecclésiastiques, de religieuses de l'Hôtel-Dieu. 
certaines caractéristiques internes. Or il apparaît que de toutes les fêtes mariales, les plus en honneur sont celles de l'ImmaculéeConception, de la Purification et de l'Assomption. Raison en est, à notre avis, qu'elles font écho à des influences ou plus tenaces ou plus suggestives. A n'en pas douter, la confrérie de la SainteFamille n'a pas peu contribué à sensibiliser les mères de famille au mytère de la Purification. L'explication de Mgr de SaintVallier vaut d'être retenue ici : ${ }^{22}$

[...] c'est une loüable coûtume de le faire [la bénédiction des femmes après leurs couches]. Cette cérémonie a été instituée afin que les femmes imitent la Très-Sainte Vierge, qui vint au Temple pour obéir à la Loy de la Purification, \& pour y presenter son Fils; afin aussi qu'elles rendent grâces à Dieu de l'heureux succès de leurs couches, \& qu'elles lui offrent le fruit qu'il lui a plû leur donner [...].

De même la coutume de commémorer chaque année, le 15 août, la consécration du royaume de France, faite par Louis XII le 10 février $1638^{23}$, a renforçi le prestige de la fête de l'Assomption. Un déploiement aussi faste que possible rassemblait tout le monde "pour reconnaître cette grande princesse comme supérieure et protectrice de l'une et l'autre France" ${ }^{24}$.

De toute évidence cependant l'image qui a les plus tenaces résonances dans la sensibilité populaire est celle de l'ImmaculéeConception. Attachement singulier qui n'a rien d'étonnant ce semble - si l'on se rappelle la mystique particulière des premiers missionnaires d'abord et reprise par Mgr de Laval ensuite. Ce symbole marial prend peu à peu l'aspect d'un bien de famille que tous partagent.

La décantation aurait commencé avec les Récollets et par leur geste de dédier leur première chapelle, en 1615, à l'Imma-

${ }^{22}$ Rituel du diocèse de Québec, 2e édition: 78. Nous citerons désormais : Rituel, et sauf indications contraires, la 2e édition.

23 Maurice Vloberg, Le vœu de Louis XIII, dans Maria, V: 519-533.

${ }^{24} \mathrm{La}$ célébration est souventes fois décrites dans le Journal des Jésuites, par exemple pour les années 1646, 1647, 1648, 1650, 1662. Voir édition Laverdière et Casgrain: 62, 93-94, 114, 142, 310. Le Père Le Jeune, à qui nous empruntons la citation, a décrit la première dans sa Relation de 1688. Voir Thwaites, op. cit., XIV : 264-265. 
culée ${ }^{25}$. Mais les vrais chefs de file qui ont appuyé la tradition mariale canadienne sont les Jésuites. Seuls autorisés à revenir en 1632 et seuls en activité jusqu'à l'arrivée des Sulpiciens en 1657 leur influence ne peut pas ne pas avoir été déterminante. Or, en ces années où les théologiens discutent ferme dans la chrétienté occidentale le privilège de l'Immaculée-Conception ${ }^{26}$, il est de tradition chez eux de la défendre et d'en propager le culte. Les missionnaires qui œuvrent en Nouvelle-France en font sans conteste le pivot de leur mystique personnelle et leur arme de conquête. Il n'en est pas meilleure preuve que le vœu de 1635. Simple geste de dévotion privée, suggéré par le Père Le Jeune, il a été prononcé le 8 décembre simultanément à Québec et en Huronie. En voici le texte tel qu'il fut consigné dans la Relation de la même année: ${ }^{27}$

[...] nous avons tous été d'avis de recourir au Ciel et à la très Sainte Vierge Mère de Dieu, par laquelle Dieu a coutume de faire ce qui ne se peut faire, et convertir les cours les plus abandonnés. A cet effet nous avons résolu de faire un vœu fort solennel, dont voici la teneur:

Mon Dieu et mon Sauveur Jésus, quoique nos péchés nous doivent éloigner de votre présence, si est-ce qu'épris d'une affection de vous honorer et votre très sainte Mère, poussé d'un désir de nous voir dans la fidèle correspondance que vous désirez de vos serviteurs, souhaitant en outre de vous voir reconnu et adoré de tous ces pauvres peuples: nous vous promettons et faisons vœu, comme aussi à la très Sainte Vierge, votre Mère, et à son glorieux époux, Saint Joseph, de célébrer douze fois es douze mois suivants, le sacrifice de la sainte messe pour ceux qui

25 A. Godbout, Les Récollets apôtres de Marie sous le Régime français, dans Soc. can. hist. Egl., rapport 1953-1954: 14. L'auteur revendique pour eux l'honneur d'avoir été les premiers propagandistes du privilège en Amérique. Le Père Adrien Pouliot infirme cette thèse dans son étude A vox origines de notre dévotion à l'Immaculée-Conception, Cahiers d'Histoire de la Société Historique de Québec - no 8 (1956) : 2-10.

26 A. Duval, o.p., La dévotion mariale dans l'ordre des frères prêcheurs, dans Maria, VI: 737-782. La célèbre dispute est racontée: 755-768.

27 Thwaites, op. cit., VIII: 190-192. Pour la commodité nous utilisons la transcription du Père Léon Pouliot, Le vœu de 1635 à l'ImmaculéeConception, dans Soc. can. hist. Egl., rapport 1953-1954: 131-132. 
sont prêtres, et pour les autres, de réciter douze fois la couronne ou chapelet de la Vierge, en l'honneur et en action de grâces de son Immaculée Conception, et de jeûner tous, la veille de cette fête; vous promettant en outre que si on érige quelque église ou chapelle stable dans ce pays, dans le cours de ce temps limité, nous la ferons dédier à Dieu sous le titre de l'Immaculée Conception, si cela est en notre pouvoir; le tout, pour obtenir de la bonté de N.S. la conversion de ces peuples, par l'entremise de sa sainte Mère et de son saint époux. Recevez cependant, ô l'Empérière des anges et des hommes, les cœurs de ces pauvres barbares abandonnés, que nous vous présentons par les mains de votre glorieux époux et de vos fidèles serviteurs $S$. Ignace et $S$. François Xavier et de tous les anges gardiens de ces misérables contrées, pour les offrir à votre Fils, afin qu'il leur donne sa connaissance et leur applique le mérite de son précieux sang. Ainsi-soit-il.

Il n'y a pas lieu d'insister ici très longuement sur la mystique qui a inspiré ce geste. Il faut néanmoins en faire remarquer le rayonnement extensif. C'est à la suite de ce vœu que la chapelle des Trois-Rivières en 1636 , celle de Sillery en 1637 , un poste de mission en Huronie en 1638 sont placés sous le vocable de l'Immaculée. L'offrande fut renouvelée chaque année, d'une façon officielle dès l'année suivante, non seulement par les missionnaires jésuites mais aussi par les Hospitalières de l'Hôtel-Dieu et les Ursulines de Québec ${ }^{28}$; le geste fut imité en 1665, à la demande de Mgr de Laval, par les prêtres et les élèves du Séminaire de Québec ${ }^{29}$. Il semble que l'évêque l'ait proposé à ses

28 Dom A. Jamet, Les Annales de l'Hôtel-Dieu de Québec, 73. Selon le Père Léon Pouliot, c'est le Père Brébeuf qui en 1641-1642 engagea les communautés à s'obliger par vœu de faire la communion mensuelle à la même intention. Soc. can. hist. Egl., rapport 1953-1954: 134.

29 Une formule adaptée du vœu de 1635 qui est renouvelé depuis, chaque année, le 8 décembre se trouve dans Mandements des Evêques, I: 67-68. Les élèves faisaient "acte de bon propos". Pour cette question, voir F. Vandry, La foi de l'Eglise de Québec en l'Immaculée-Conception au temps de Mgr de Laval, dans Soc. can. hist. Egl., rapport 1953-1954: 31ss. Quant à Mgr de Laval et sa dévotion à l'Immaculée, voir Sacra Rituum Congregatio - Sectio Historica, Quebecen Beatificationis et Canonizationis ven. servi Dei Francisci de Montmorency Laval, altera nova positio. MCMLVI : xcix. 
diocésains lors de la consécration de sa cathédrale en $1666^{30}$. Quoi qu'il en soit la Relation de 1637 donne à entendre que les colons n'ont pas attendu l'invite du prélat pour honorer de façon plus particulière le privilège marial ${ }^{31}$. On peut croire que la première 'congrégation' mariale, placée sous le même vocable, fut un effet de leur dévotion à l'Immaculée.

Bénéficiaires de la direction spirituelle des Pères jésuites ${ }^{32}$, les religieuses de Québec étaient à même de prolonger leur influence auprès des malades et des enfants commis à leurs soins. Il n'empêche que les Ursulines pratiquaient une affection bien particulière pour "Notre Dame de Grand Pouvoir" qu'elles considéraient comme la première supérieure de leur couvent ${ }^{33}$ et que les Hospitalières de l'Hôtel-Dieu honoraient très spécialement "Notre Dame de Protection" ${ }^{34}$. Voisins, ces vocables faisaient

${ }^{30}$ Léon Pouliot, op. cit.: 136.

31 Thwaites, op. cit., XII : 76. Au milieu de son récit le Père Le Jeune laisse tomber cette réflexion: "[...] la veille de la Conception de la saincte Vierge, que nous honorons fort en la nouvelle France [...]".

32 D'après une pièce en montre au musée des Ursulines de Québec, le Père J. Lalemant a écrit avec Marie de l'Incarnation les constitutions du monastère qui ont été en vigueur de 1647 à 1681. - On peut voir une liste des confesseurs des religieuses de l'Hôtel-Dieu dans Histoire de l'Hôtel-Dieu de Québec par H. R. Casgrain, 572-573 (appendice).

83 Cette pratique aurait commencé à Tours en 1632. Chaque année, à partir de 1650, les religieuses renouvelaient le 8 septembre leur acte d'obéissance à Notre-Dame. Les Ursulines de Québec depuis leur établissement jusqu'à nos jours, I: 149. de Québec.

34 Une statue de l'époque se trouve aujourd'hui à l'Hôpital Général

Nous croyons de voir signaler ici, parce que c'est le point de départ d'une nouvelle lancée mariale en Nouvelle-France, la dévotion au Cour Immaculée de sœur Catherine de St-Augustin. Conquise à cette dévotion par le Père Eudes lui-même (ACNDM, Notes sur divers sujets, no 2: 63) Catherine de Longpré (1632-1668) était une compatriote du missionnaire (voir H.-R. Casgrain, op. cit.: 150ss). Devenue maîtresse des novices le 9 novembre 1665 elle en profita pour "initier ses religieuses à cette forme nouvelle du culte marial" (Henri Cormier, Origines de la dévotion au Cœur Immaculé de Marie au Canada, dans Soc. can. hist. Egl., rapport 1953-1954: 103). Mais à l'époque où nous sommes il ne s'agissait alors que d'une dévotion privée dont le rayonnement, pour autant que l'on sache, ne dépassait pas le groupe des religieuses de l'Hôtel-Dieu. Ce culte particulier prendra ses dimensions populaires sous l'épiscopat de Mgr de Saint-Vallier.

Quant à 'l'esclavage' marial pratiqué par Marie de l'Incarnation, il n'apparaît pas avoir beaucoup rayonné en dehors d'un cercle restreint de quelques âmes d'élite, dont en particulier le Père J.-M. Chaumonot. La 
même appel à la puissance tutélaire de Marie, de la même façon que "Notre Dame de Pitié", invoquée par les Hospitalières de Saint-Joseph à l'Hôtel-Dieu de Montréal ${ }^{35}$.

A Ville-Marie en effet, durant la même période, la pieuse Marguerite Bourgeoys poursuit son œuvre et ne laisse pas de promouvoir au niveau populaire le culte de Notre-Dame. On sait dans quel imbriquement spirituel elle se trouve mêlée; Olier, La Dauversière, la Société Notre-Dame de Montréal en appellent eux aussi à l'Immaculée ${ }^{36}$. Sans déroger, mais femme pratique avant tout, elle vise à faire poser des gestes de confiance engageants. Dans le concret cela signifie la construction en corvée de la chapelle Notre-Dame-de-Bon-Secours ${ }^{37}$, l'établissement, le 3 juillet 1658, sous le patronage de Notre-Dame-de-la-Victoire d'une 'congrégation externe' pour jeunes filles "dont l'éducation était terminée" ${ }^{38}$, la première association de la Sainte-Famille fondée avec le concours de Gabriel Souart, sulpicien, et de sœur Catherine Macé ${ }^{39}$. Pour assurer une milice défensive permanente il se fonde, le 27 janvier 1663 , une association de "soldats de la Vierge" ${ }^{40}$. Les Sulpiciens de leur côté, arrivés depuis peu, zèlent

dévotion singulière de l'Ursuline ne saurait faire de doute. Dans une lettre à son fils en effet, en date du 16 septembre 1661, elle confie: "Je porte au cou une petite chaîne de fer depuis plus de vingt-trois ans, pour marque de mon engagement à la sainte Mère de Dieu; je n'y ai point d'autre pratique, sinon, en la baisant, de m'offrir pour esclave à cette divine Mère" (abbé Richaudeau, Lettres de la révérende mère Marie de l'Incarnation, II: 200). Il est curieux que l'étude du Père Henri de Lubac, Marie de l'Incarnation et la Sainte Vierge, dans Maria, III: 181-204, ait passé sous silence ce détail important. Compte tenu de l'ensemble du mouvement marial de l'époque en Nouvelle-France, cette forme personnelle de dévotion ne constitue pas à notre avis une caractéristique du climat marial qui fait l'objet du présent chapitre.

35 AHDM, Année mariale 1954: Centenaire du dogme de l'ImmaculéeConception: 10ss.

36 AHDM, ibid.: 1ss.

37 AHDM, Annales de Soeur Morin: 70 (74).

38 E.-M. Faillon, Vie de la Saur Bourgeois [...], I: 185.

39 AHDM, Annales, III: 81. Rappelons que depuis 1656 la chapelle de l'Hôtel-Dieu servait d'église paroissiale. Voir Sœur M. Mondoux, L'HôtelDieu premier hôpital de Montréal, 135; E.-M. Faillon, Vie de Mademoiselle Mance [...], I: 233.

40 AHDM, Année mariale 1954: Centenaire du dogme de l'ImmaculéeConception, 8. L'auteur cite un chiffre: 63 qu'elle dit emprunter à Sœur Morin. G. Lanctot, op. cit.: 40, parle de 140. 
dans le sens de la spiritualité de leur fondateur. Leur première église paroissiale, édifiée en 1672, est dédiée à la Purification, fête patronale de l'Institut et de la Société Notre-Dame. Une chapelle iroquoise construite dans la montagne en 1680 sera placée sous le vocable de Notre-Dame-des-Neiges ${ }^{41}$.

Ainsi à l'arrivée du premier évêque de Québec, le 16 juin 1659 , le terroir religieux est donc fortement marqué d'une tradition mariale. Bien loin de se contenter d'en être le témoin le plus digne, Mgr de Laval s'emploie lui aussi à l'enraciner davantage. Surtout en ce qu'elle touche à l'Immaculée-Conception dont on peut dire qu'il en fait le premier centre de son activité apostolique. Pour ne relever que les signes les plus proches ${ }^{42}$ : un choix délibéré du 8 décembre (1658) pour recevoir la consécration épiscopale, une reprise personnelle - en 1665 - du vœu de 1635, mise sous patronage spécial de l'Immaculée de tout son diocèse (1666). Il a déjà par ailleurs loué le culte du très pur Cœur de Marie ${ }^{43}$. Le même souci de faire honorer la Vierge d'une façon spéciale se retrouve dans le règlement qu'il édicte pour les élèves du petit séminaire ${ }^{44}$, ou encore dans les statuts de la Confrérie de la Sainte-Famille qui semble avoir été la préférée de son cœur ${ }^{45}$. Pour reprendre l'expression de son panégyriste, il avait "une confiance particulière en la Mère Dieu

41 G. Lanctot, op. cit.: 45; Sœur Mondoux, op. cit.: 136.

42 Ancien congréganiste du collège de La Flèche, un consacré à la Vierge depuis le 2 février 1634, membre de la "Société des bons amis" (1644) avec son ami intime Henri-Marie Boudon et un pieux laĩque du nom de Jean de Bernières, il est un conquis de longue date. Nouvellement consacré évêque, il réside à l'Ermitage de Caen où il noue une solide amitié avec le Père Jean Eudes; c'est de là qu'il part pour la Nouvelle-France. Voir H. Cormier, op. cit.: 99-109; Sacra Rituum Congregatio, op. cit.: xcviii, 218-219.

43 Lettre du 22 décembre 1663 dans Sacra Rituum Congregatio, op. cit.: xcix.

44 Mention a déjà été faite de l' "Acte de bon propos". Sont prévus en outre la récitation quotidienne du Petit Office de l'Immaculée ou du chapelet, et à l'intention des missionnaires, de l'Ave Maris Stella, du Salve Regina le samedi, un pèlerinage annuel à Notre-Dame-de-Foy; même en vacances, récitation quotidienne du Petit Office ou du chapelet. Il $y$ avait aussi deux 'congrégations' dédiées à l'Assomption pour les internes et à l'Immaculée pour les externes. Amédée Gosselin, L'Instruction au Canada sous le régime français, 419, 424, 439.

45 Mandements des Evêques, I: 56ss. 
conçue sans péché. Car en fait de dévotion à la Sainte Vierge [...] jamais prélat n'a eu plus de soin de faire honorer cette Reine des Anges dans son diocèse et d'en inspirer l'amour à ses diocésains." 46

La litanie des titres marials évoqués ci-dessus diffère bien quelque peu selon les lieux et les artisans. Si la lointaine Acadie montre un attachement plus particulier pour Notre-Dame de l'Assomption ${ }^{47}$, ailleurs un accent majeur est mis sur le privilège de l'Immaculée-Conception. Sur le plan de la dévotion les vocables utilisés n'ont de soi rien d'exclusif. Ce qu'il importe de retenir c'est l'orientation foncière qu'ils expriment, ce sont les modalités du culte qui s'y rattache. Au terme de cette période précédant l'arrivée de Mgr de Saint-Vallier, la tendance commune de la dévotion mariale en Nouvelle-France est de prendre appui sur le patronage de Marie. C'est là un trait caractéristique de la collectivité canadienne. Que l'Immaculée soit devenue le premier - mais non le seul symbole d'appel à la confiance -, cela tient à l'influence décisive des Pères jésuites appuyés par Mgr de Laval ${ }^{48}$; cela tient aussi, à ce qu'il semble, au fait que le plus fort groupe de colons immigrés au pays durant la même période venaient de cette Normandie qui "de toutes les provinces françaises semble avoir été la plus dévote à l'Immaculée-Conception" ${ }^{49}$.

Quoi qu'il en soit, un climat marial existe profond, actif. Les interactions des hommes et les circonstances ont fait qu'à l'arrivée de Mgr de Saint-Vallier. Notre-Dame fait partie des

46 Oraison funèbre par Joseph de la Colombière, AAQ, Actes de sépulture - Oraisons funèbres et Testaments des Evêques de Qwébec: 11.

47 Théodore Gallant, Dévotion à Notre-Dame de l'Assomption en Acadie, dans Soc. can. hist. Egl., rapport 1953-1954: 91-97.

48 Pour mieux s'en convaincre, il n'est que de relire l'étude du Père Adrien Pouliot, Aux origines de notre dévotion à l'Immaculée-Conception, Cahiers d'Histoire de la Société historique de Québec — no 8 (1956) : 11-28.

49 Jean-Léon Allie, o.m.i., La Nouvelle-France et les premiers monuments de foi en l'Immaculée-Conception, dans RUO, XII, 4 (oct.-déc. 1942) : 494-513. Citation: 509. En note l'auteur fournit des chiffres: des 3802 immigrants venus en 1608 et 1680,1038 venaient de Normandie où "la légende fait instituer pour la première fois dans le royaume marial la fête de la Conception". 
attentions spirituelles et de la vie de l'Église canadienne. A partir de la tête jusqu'au bas de la société. ${ }^{50}$

HeCTOR BIBEAU, s.m.m.

50 Sans trop presser les statistiques pour leur faire dire plus qu'elles ne contiennent, il est intéressant d'avoir au moins un ordre de grandeur de la communauté canadienne. Selon les chiffres du recensement du Canada pour l'année 1685, la jeune Eglise comptait alors 107 membres du clergé, soit: 38 prêtres, 14 ecclésiastiques, 43 Jésuites et 11 Récollets, et 80 religieuses dont 28 Ursulines, 26 Hospitalières à Québec et 16 à Montréal, 10 filles dévotes en congrégation pour 10725 Français et 1438 sauvages. Ces chiffres sont publiés dans J. Blanchet, op. cit., 350 . 